\title{
MicroRNA in a Case of Unexplained Recurrent Pregnancy Loss
}

\section{Heba Al-Shorafa and Fadel A Sharif*}

Department of Medical Laboratory Sciences, Islamic University of Gaza, Gaza, Palestine

\begin{abstract}
The present report describes the relative quantitation of a group of selected miRNAs in a twenty one years old Palestinian woman who experienced 8 unexplained first trimester pregnancy losses. The relative expression of miR-21, miR-126, miR-155, miR-182, miR-222 and miR-517* in her plasma was measured before pregnancy and in the seventh week of her ninth pregnancy. The relative expression of the same miRNAs was also measured before pregnancy and in the eighth week of fifth pregnancy in a healthy control woman who has four live children and without any history of pregnancy loss.

The results of the case woman showed an extremely decreased level of all the investigated microRNAs during her pregnancy as compared to their level before being pregnant, except for miR-517* which was elevated. The relative quantitation of miR-21, miR-126, miR-155, miR-182, miR-222 and miR-517* was 0.026, 0.12, 0.23, 0.26, 0.28 and 1.4 , respectively. However, the relative quantitation of the respective miRNAs in the control was $0.92,0.75,0.6,1.1,1.11$ and 10.9. Unfortunately, at the end of eighth week of her pregnancy the case woman lost her fetus.

We believe that the dysregulation of miRNA is involved in the recurrent pregnancy losses in the case presented in this report.
\end{abstract}

Keywords: miRNA; Recurrent pregnancy loss

\section{Introduction}

MicroRNAs (miRNAs) are small ( 22 nucleotides long), single stranded, non-coding RNA molecules that bind their cognate target mRNAs to prevent protein production by one of two distinct mechanisms: translation inhibition or degradation of mRNA. Mature miRNA is generated through a two-step cleavage of precursor miRNA (pre-miRNA), where RNA-induced silencing complex (RISC) is involved in the process [1]. Currently, around 700 different miRNAs are known to exist in humans that are estimated to regulate greater than $60 \%$ of protein encoding genes $[2,3]$.

Recurrent pregnancy loss (RPL) has been historically defined as three or more consecutive pregnancy losses before 20 weeks of gestation. An estimated $1 \%$ of couples attempting pregnancy suffer three or more consecutive losses, and as many as $5 \%$ have two or more consecutive losses [4]. Causes of RPL can be categorized as genetic abnormalities, hormonal and metabolic disorders, uterine anatomic abnormalities, infectious causes, autoimmune disorders, thrombophilic disorders, alloimmune causes, and idiopathic. This latter group accounts for over $50 \%$ of cases [5].

Careful search of the literature showed that no targeted microRNA expression profiling has been performed for RPL. There are however, reports on placenta-specific miRNAs capable of crossing the placental barrier and are detectable in maternal plasma [6,7] and an altered profile of several miRNAs has been shown in pregnancy complications [8].

\section{Case Presentation}

A 21 year old housewife presented to the Genetics lab at the Islamic University of Gaza suffering from 8 unexplained RPLs. She was not pregnant at the first visit to our lab. Her husband is not her family relative. After having the couple's written informed consent and the approval of the local ethics committee, $4 \mathrm{~mL}$ peripheral blood was collected from the woman into an EDTA tube. To harvest cellfree plasma, whole blood sample was centrifuged twice at $1200 \times \mathrm{g}$ for $10 \mathrm{~min}$ at room temperature. RNA was extracted immediately after sample collection using miRNeasy RNA isolation kit (Qiagen, USA), according to the manufacturer protocol. Each miRNA (miR-21, miR126, miR-155, miR-182, miR-222, miR-517*) was reverse transcribed into complementary DNA (cDNA) using a TaqMan miRNA Assay (Applied Biosystems, USA) containing miRNA-specific stem-loop RT primers and a TaqMan MicroRNA Reverse Transcription Kit (Applied Biosystems, USA) in a total reaction volume of $15 \mu \mathrm{L}$, and was carried out according to the manufacturer's instructions. Reverse transcriptase reactions were performed using a 7500 real-time PCR system (Applied Biosystems) and the following thermal cycling parameters: $30 \mathrm{~min}$ at $16^{\circ} \mathrm{C}, 30 \mathrm{~min}$ at $42^{\circ} \mathrm{C}, 5 \mathrm{~min}$ at $85^{\circ} \mathrm{C}$, and then held at $4^{\circ} \mathrm{C}$.

Three micro liters of cDNA, corresponding to each selected microRNA, and $3 \mu \mathrm{L}$ of cDNA corresponding to RNU6B (endogenous control) were mixed with specific primers, the TaqMan MGB probe (TaqMan MicroRNA Assay; Applied Biosystems) and the ingredients of the TaqMan Universal PCR Master Mix (Applied Biosystems) in a total reaction volume of $20 \mu \mathrm{L}$. TaqMan PCR conditions were as described in the TaqMan guidelines. The analysis was performed using a 7500 real-time PCR system (Applied Biosystems).

The same protocol was applied concurrently on a blood sample of a healthy volunteer who had four live births and without any previous history of abortion. Her plasma miRNAs were also investigated before being pregnant and during her eighth week of pregnancy. All PCR reactions were performed in triplicates.

In each sample the relative amount of miRNA was calculated using the comparative threshold method determining RNU6B as the endogenous control with $\Delta \mathrm{Ct}=\mathrm{Ct}$ (miRNA)-Ct (RNU6B). Relative quantification of miRNA expression was calculated with the $2^{-\Delta \Delta \mathrm{Ct}}$ method

*Corresponding author: Fadel A Sharif, Department of Medical Laboratory Sciences, Islamic University, Gaza, Palestine, Tel: +970-59-308364; E-mail: fsharif@iugaza.edu.ps

Received November 24, 2012; Accepted December 28, 2012; Published December 31, 2012

Citation: Al-Shorafa H, Sharif FA (2012) MicroRNA in a Case of Unexplained Recurrent Pregnancy Loss. J Clin Case Rep 2:238. doi:10.4172/21657920.1000238

Copyright: (c) 2012 Al-Shorafa H, et al. This is an open-access article distributed under the terms of the Creative Commons Attribution License, which permits unrestricted use, distribution, and reproduction in any medium, provided the original author and source are credited. 
Analyses on case miRNAs were done before and during the seventh week of pregnancy. The results showed an extreme decline in all the investigated microRNAs during her pregnancy compared to her status before being pregnant except for $\mathrm{miR}-517^{\star}$ which was elevated during her pregnancy. The relative quantitation of miR-21, miR-126, miR155, miR-182, miR-222 and miR-517* was $0.026,0.12,0.23,0.26,0.28$ and 1.4 , respectively. The relative quantitation of the same miRNAs in the healthy control was $0.92,0.75,0.6,1.1,1.11$ and 10.9 , respectively. Figures 1 and 2 illustrate the fold change comparison of the selected microRNAs in the study case and control, respectively. Unfortunately, at the end of eighth week of her pregnancy the case woman lost her fetus.

\section{Discussion}

The establishment of a successful pregnancy involves a regulated interplay of several components that are contributed by both the fetus and the mother. Perhaps the most critical post-implantation event in the first trimester is the formation and maintenance of a healthy placenta. Placentation also is a phenomenon that requires the interaction of several feto-maternal cellular activities and gene products. Inflammation, cell growth and proliferation, apoptosis and differentiation, cell invasion, angiogenesis, release of various cytokines, hormones and growth factors, tuning of maternal innate and adaptive immune responses are among the prominent activities occurring during placentation. Obviously these multiple interconnected events need fine regulation of a myriad of genes. Therefore, miRNAs seem an attractive candidate for this purpose since a given miRNA has the

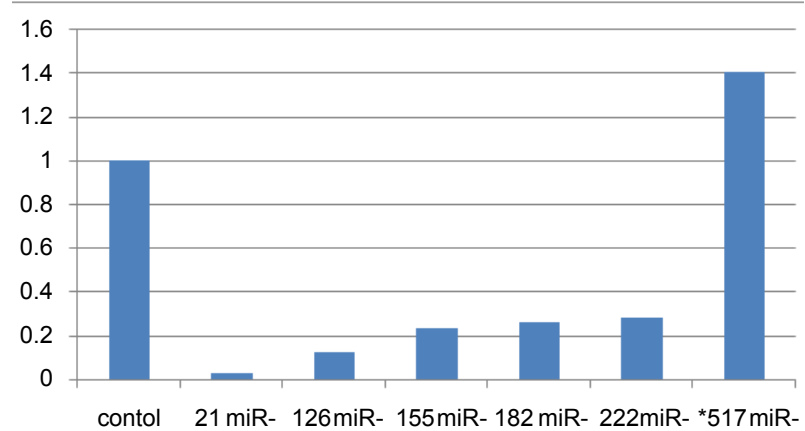

Figure 1: Fold change comparison of the selected miRNAs expression in the study case between her status before and during pregnancy, the control column represents her status before being pregnant.

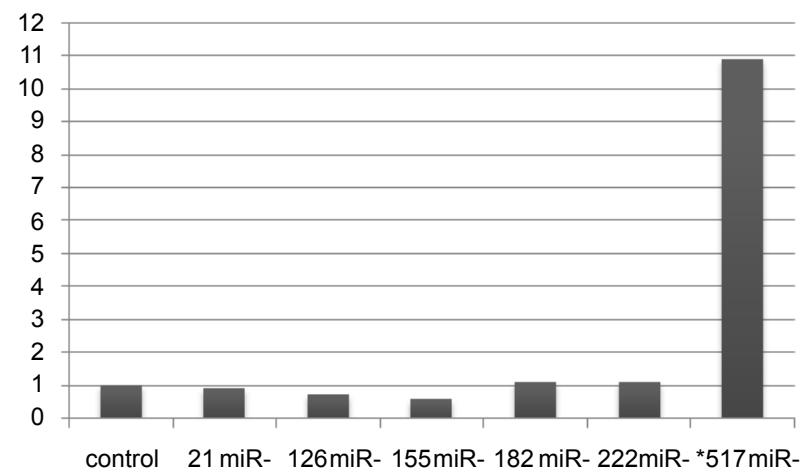

Figure 2: Fold change comparison of the selected miRNAs expression in the control subject between her status before and during pregnancy, the control column represents her status before being pregnant. ability to regulate and thus coordinate the expression of many genes simultaneously [9].

Several lines of evidence make us believe in the involvement of miRNAs in regulating and coordinating the plethora of gene activities needed during pregnancy. First, the existence of placenta- and trophoblast-specific miRNAs and the ability of those miRNAs to find their way to the maternal circulation $[6,7,9,10]$. Second, deviations from the normal expression pattern of miRNAs likely play role in many diseases such as cancer, cardiovascular diseases and pre-eclampsia [1113]. Third, the potential of miRNAs to act as signaling molecules i.e., miRNAs released by certain cells can be taken up by other cells where they can elicit their regulatory effects [14]. Finally, Renthal et al. [15] demonstrated that certain miRNAs can modulate uterine quiescence and contractility during pregnancy and labor.

The miRNAs investigated in the present work were chosen as they are known to regulate many biological events that we think are crucial in pregnancy. For instance, miR-21 was selected because it promotes cellular proliferation, migration and invasion [16]. Moreover, miR21 has also been shown to be taken up by macrophages through Toll-like receptor 8 where it induces the release of cytokines that promote inflammation [14]. miR-126 was chosen because of its role in angiogenesis. This miRNA is a key regulator of vascular endothelial growth factor and fibroblast growth factor signaling in endothelial cells [17].

miR-155 was included as it is involved in inflammatory response and homeostasis of many immune cells [2]. miR-182 was selected because of its role in regulating cell proliferation [18]. miR-222 is involved in regulation of angiogenesis [19]. miR-517* was included because it is placenta-specific and has been shown to be highly expressed in maternal circulation especially at the first trimester [9].

The relative quantitation of the investigated miRNAs (miR-21, miR126, miR-155, miR-182, miR-222) showed a sharp decrease in the RPL case during her pregnancy as compared to their level before pregnancy. However, the level of the same miRNAs was not appreciably changed before and after pregnancy in the healthy control. Even during their pregnancy, the placenta-specific miR-517 ${ }^{\star}$ was unexpectedly lower in the RPL as compared to the healthy control (1.4 vs. 10.9 fold).

Overall the results indicate that pregnancy in the RPL case somehow affected the release of normal levels of all investigated miRNAs (and perhaps other miRNAs), with consequent abnormality in all downstream processes regulated by those miRNAs. Repression of production or release of miRNAs by an undetermined pregnancyrelated effector may be the cause of the decline in miRNAs level in the RPL case. In support of this hypothesis studies have shown that leukemia inhibitory factor can reduce the level of certain miRNAs and can alter miRNome of trophoblastic cells [19]. Additionally, the release of miRNAs has been shown to be controlled by neutral sphingomyelinase-2 and through ceramide-dependent secretory machinery [20].

We believe that aberrant expression of miRNAs is the cause of recurrent abortion in the RPL case. Further work is needed in order to find pregnancy-associated factor(s) involved in miRNA homeostasis during pregnancy.

\section{Acknowledgement}

This study was supported by a grant from the Scientific Research Affairs at the Islamic University-Gaza, 2011-2012 annual research grants. 


\section{References}

1. Huntzinger E, Izaurralde E (2011) Gene silencing by microRNAs: contributions of translational repression and mRNA decay. Nat Rev Genet 12: 99-110.

2. O'Connell RM, Rao DS, Chaudhuri AA, Baltimore D (2010) Physiological and pathological roles for microRNAs in the immune system. Nat Rev Immunol 10: 111-122

3. Bartel DP (2004) MicroRNAs: genomics, biogenesis, mechanism, and function. Cell 116: 281-297.

4. Coulam CB (1991) Epidemiology of recurrent spontaneous abortion. Am J Reprod Immunol 26: 23-27.

5. Lee RM, Silver RM (2000) Recurrent pregnancy loss: summary and clinical recommendations. Semin Reprod Med 18: 433-440.

6. Chim SS, Shing TK, Hung EC, Leung TY, Lau TK, et al. (2008) Detection and characterization of placental microRNAs in maternal plasma. Clin Chem 54 482-490.

7. Chiu RW, Lo YM (2010) Pregnancy-associated microRNAs in maternal plasma: a channel for fetal-maternal communication? Clin Chem 56:1656-1657.

8. Morales Prieto DM, Markert UR (2011) MicroRNAs in pregnancy. J Reprod Immunol 88: 106-111.

9. Kotlabova K, Doucha J, Hromadnikova I (2011) Placental-specific microRNA in maternal circulation--identification of appropriate pregnancy-associated microRNAs with diagnostic potential. J Reprod Immunol 89: 185-191.

10. Morales-Prieto DM, Chaiwangyen W, Ospina-Prieto S, Weber M, Weise A, et al. (2012) The miRNome of trophoblast cells. J Reprod Immunol 94: 18-19.

11. Calin GA, Croce CM (2006) MicroRNA signatures in human cancers. Nat Rev Cancer 6: 857-866.
12. van Rooij E, Olson EN (2007) MicroRNAs: powerful new regulators of heart disease and provocative therapeutic targets. J Clin Invest 117: 2369-2376.

13. Noack F, Ribbat-Idel J, Thorns C, Chiriac A, Axt-Fliedner R, et al. (2011) miRNA expression profiling in formalin-fixed and paraffin-embedded placental tissue samples from pregnancies with severe preeclampsia. J Perinat Med 39: $267-$ 271.

14. Fabbri M, Paone A, Calore F, Galli R, Gaudio E, et al. (2012) MicroRNAs bind to Toll-like receptors to induce prometastatic inflammatory response. Proc Natl Acad Sci U S A 109: E2110-116.

15. Renthal NE, Chen CC, Williams KC, Gerard RD, Prange-Kiel J, et al. (2010) MiR-200 family and targets, ZEB1 and ZEB2, modulate uterine quiescence and contractility during pregnancy and labor. Proc Natl Acad Sci U S A 107: 2082820833.

16. Lou Y, Yang X, Wang F, Cui Z, Huang Y (2010) MicroRNA-21 promotes the cell proliferation, invasion and migration abilities in ovarian epithelial carcinomas through inhibiting the expression of PTEN protein. Int J Mol Med 26: 819-827.

17. Zernecke A, Bidzhekov K, Noels H, Shagdarsuren E, Gan L, et al. (2009) Delivery of microRNA-126 by apoptotic bodies induces CXCL12-dependent vascular protection. Sci Signal 2: 81.

18. Kong WQ, Bai R, Liu T, Cai CL, Liu M, et al. (2012) MicroRNA-182 targets cAMP-responsive element-binding protein 1 and suppresses cell growth in human gastric adenocarcinoma. FEBS J 279: 1252-1260.

19. Morales-Prieto DM, Schleussner E, Markert UR (2011) Reduction in miR141 is induced by Leukemia Inhibitory Factor and Inhibits Proliferation in Choriocarcinoma Cell Line JEG-3. Am J Reprod Immunol 66: 57-62.

20. Kosaka N, Iguchi H, Yoshioka Y, Takeshita F, Matsuki Y, et al. (2010) Secretory mechanisms and intercellular transfer of microRNAs in living cells. J Biol Chem 285: $17442-17452$ 